\title{
Цифровая трансформация
}

\section{производственных процессов и бизнес-моделей горнодобывающей промышленности в условиях рыночной нестабильности}

\section{САВОН Д.Ю.}

Доктор экон. наук, профессор кафедры «Промышленный менеджмент» НИТУ «МИСиС», 119049, г. Москва, Россия, e-mail:di199@yandex.ru

\section{ШКАРУПЕТА E.B.}

Доктор экон. наук, профессор кафедры «Цифровая и отраслевая экономика» ФГБОУ ВО «Воронежский государственный технический университет», 394071, 2. Воронеж, Россия, e-mail:9056591561@mail.ru

\section{САФРОНОВ А.Е.}

\section{Доктор экон. наук,}

профессор кафедры «Менеджмент и бизнес-технологии» ФГБОУ ВО «Донской государственный

технический университет»,

344010, г. Ростов-на-Дону, Россия,

e-mail: reception@dstu.edu.ru

\author{
АНИСимов А.Ю. \\ Канд. экон. наук, \\ дочент кафедры «Финансы и кредит» \\ НАНО ВО «Институт мировых чивилизачий», \\ 119991, г. Москва, Россия, \\ e-mail:anisimov_au@mail.ru
}

\section{ВИХРОВА Н.О.}

Канд. экон. наук,

дочент кафедры «Экономика»

НИТу «МИСиС»,

119049, г. Москва, Россия,

e-mail: natalia.vichrova@yandex.ru
Цифровая трансформация производственных процессов и бизнес-моделей горнодобывающих предприятий, идущая рука об руку с выбором соответствующих цифровых технологий, сможет обеспечить долгосрочные решения тревожных экономических проблем нашего времени. В статье поднимаются вопросы, связанные с текущим и будущим состоянием горнодобывающей промышленности до, во время и после цифровой трансформации. В ходе исследования рассмотрены: необходимость, преимущества, содержание, цели и задачи, принципы, основные направления, инструменты и этапы осуществления цифровой трансформации горнодобывающих предприятий. Выделены системные проблемы цифровой трансформации отрасли, на основе решения которых авторами предложен алгоритм цифровой трансформации горнодобывающих предприятий с пошаговым внедрением, который уже начал применяться в холдинге «ЕВРАЗ». В процессе цифровой трансформации особая роль отведена руководителю цифровой трансформации (CDTO). Представленные разработки позволят комплексно преобразовать отечественную горнодобывающую промышленность на основе изменения стратегии и применения новых операционных бизнес-моделей на глобальном уровне в условиях рыночной нестабильности, волатильного спроса, проблем поиска новых месторождений в новых регионах, необходимости более длительной эксплуатации активов, ориентации на высокие стандарты в работе, а также изменений в правилах ведения горнодобывающего бизнеса по всему миру.

Ключевые слова: чифровая трансформачия, иифровизация, горнодобывающая промышленность, горные предприятия, горнодобывающие предприятия, горнодобывающая отрасль, руководитель иифровой трансформачии, СDTO.

Для цитирования: Цифровая трансформация производственных процессов и бизнес-моделей горнодобывающей промышленности в условиях рыночной нестабильности / Д.Ю. Савон, Е.В. Шкарупета, А.Е. Сафронов и др. // Уголь. 2021. № 2. С. 32-37. DOI: 10.18796/0041-57902021-2-32-37. 


\section{ВВЕДЕНИЕ}

Горнодобывающая промышленность стоит на пороге радикальных структурных изменений, вызванных цифровой трансформацией всех процессов. Цифровизация - необходимое условие для устойчивого роста производительности и доходности, сохранения конкурентного преимущества.

Цифровая трансформация несет в себе как огромный потенциал, так и серьезные вызовы. При цифровой трансформации происходит резкое снижение транзакционных издержек за счет появления новых моделей деятельности, прежде всего цифровых платформ; соединение возможностей технологий и традиционной сферы деятельности организации приводит к появлению новых продуктов и процессов с принципиально иными качествами.

\section{ЭВОЛЮЦИЯ ЦИФРОВОЙ ТРАНСФОРМАЦИИ ГОРНОДОБЫВАЮЩЕЙ ПРОМЫШЛЕННОСТИ}

Рассматривая эволюцию цифровой трансформации горнодобывающей промышленности, можно выделить три этапа ее осуществления: автоматизация $\rightarrow$ цифровизация $\rightarrow$ цифровая трансформация.

На первом этапе, который большинство горнодобывающих предприятий уже почти завершили, должна быть осуществлена автоматизация в части внедрения информационных технологий. Причем для автоматизации характерно внедрение IT-решений, повторяющих имеющиеся процессы. Предыдущие попытки внедрения информационных технологий в горнодобывающей промышленности привели к созданию разрозненных информационных систем и часто дублировали, не изменяя, «бумажные» процессы [1].

Вторым этапом, происходящим в настоящее время, является цифровизация горнодобывающих предприятий, то есть приход в горную промышленность не просто информационных, а именно цифровых технологий, которые радикально удешевят производство. По результатам исследования, проведенного компанией KPMG в 2019 г., ключевыми цифровыми технологиями в России стали следующие восемь: роботизация (RPA), анализ больших данных (Big Data) и предиктивная аналитика, чат-боты, искусственный интеллект (Al), виртуальная и дополненная реальность VR/AR, интернет вещей (IoT), оптическое распознавание и блокчейн. Цифровые технологии внедряются в горнодобывающей промышленности в условиях Индустрии 4.0.

Третьим этапом в эволюции цифровой трансформации должно стать внесение коренных изменений в технологии, культуру, операции и принципы создания новых продуктов и услуг. Индустрия 4.0 всего лишь часть более глобального процесса. Сегодня уже говорят о рождении суперинтеллектуального социума - Общества 5.0 [2, 3, 4, 5]. Заглядывая вперед, можно предположить, что скоро появится и Индустрия 5.0, которая, по мнению авторов, будет ориентирована не на цифровую трансформацию, а на коммуникацию людей и созданных цифровых технологий.

\section{НЕОБХОДИМОСТЬ И ПРЕИМУЩЕСТВА ЦИФРОВОЙ ТРАНСФОРМАЦИИ ГОРНОДОБЫВАЮЩИХ ПРЕДПРИЯТИЙ}

Проблема эффективного использования цифровых технологий в горнодобывающем секторе является чрезвычайно актуальной. Нами были проанализированы трудно- сти, с которыми сталкивается горнодобывающая отрасль, и потенциальные преимущества использования цифровых технологий для преодоления существующих вызовов (табл. 1).

Существующий опыт реализации проектов по автоматизации и цифровизации, программ цифровой трансформации и Индустрии 4.0 в горнодобывающей промышленности систематизирован и представлен в табл. 2.

Помимо кейсов, рассмотренных в табл. 2, заслуживают отдельного внимания разработки компании $\mathrm{AO}$ «ВИСТ Групп» по созданию Интеллектуального Карьера. Добывающие компании, которым при помощи модели Цифрового Карьера удастся вывести эффективность работы оборудования на уровень, близкий к уровню эффективности в секторе промышленного производства, займут лидирующие позиции на рынке. По мнению авторов, ключевыми технологиями в перспективе 5-10 лет на горнодобывающих предприятиях станут цифровые двойники. Так, в 2019 г. ИВТ СО РАН выполнена пилотная реализация программной части цифрового двойника очистного забоя угольной шахты $[9,10,11]$.

\section{АЛГОРИТМ РЕАЛИЗАЦИИ ПРОГРАММЫ ЦИФРОВОЙ ТРАНСФОРМАЦИИ ГОРНОДОБЫВАЮЩИХ ПРЕДПРИЯТИЙ НА ОСНОВЕ ВОЛНОВОГО ПОДХОДА}

Для эффективной цифровой трансформации горнодобывающих предприятий возможно использовать так называемый «волновой» подход. Мы рекомендуем разработать комплексную программу цифровой трансформации (дорожную карту, подробный сценарий реализации стратегии цифровой трансформации), реализуемую по принципу перекрывающих друг друга «волн», каждая из которых превосходит предыдущую по сложности и полезному эффекту (см. рисунок). Это позволит объединить различные инициативы в области цифровизации в целостную стратегию.

Алгоритм реализации программы цифровой трансформации горнодобывающих предприятий включает в себя последовательное выполнение четырех этапов из шести шагов. Цифровая трансформация горнодобывающих предприятий возможна только при новом подходе к ролям в командах и появлении команд цифровой трансформации. По данным PwC, только в семи горнодобывающих компаниях из мирового ТОП-40 в составе высшего руководства есть директор по технологиям, директор по ИТ и директор по цифровым технологиям. Поэтому первоочередная задача в рамках начала осуществления цифровой трансформации горнодобывающей промышленности - назначение CDTO с наделением его соответствующими функциями и полномочиями и формирование Центра компетенций. CDTO - проводник единой политики цифрового развития обеспечивает управление реализацией проектов цифровой трансформации; согласовывает стратегические документы и бюджеты по информатизации и цифровой трансформации; осуществляет координацию структурных подразделений по цифровой трансформации и цифровому развитию; организовывает управление данными; формирует Центр компетенций, выполняет иные задачи. 


\section{Трудности и потенциальные преимущества использования цифровых технологий в горнодобывающей отрасли}

\begin{tabular}{|c|c|}
\hline $\begin{array}{l}\text { Трудности, с которыми } \\
\text { сталкивается отрасль }\end{array}$ & $\begin{array}{c}\text { Потенциальные преимущества использования } \\
\text { цифровых технологий }\end{array}$ \\
\hline $\begin{array}{l}\text { Эффективная реализация проектов } \\
\text { капитальных вложений }\end{array}$ & $\begin{array}{l}\text { Осуществление проектов в сроки и в рамках выделенного бюджета } \\
\text { Эффективные методы работы с подрядчиками }\end{array}$ \\
\hline $\begin{array}{l}\text { Недозагрузка производственных } \\
\text { мощностей }\end{array}$ & $\begin{array}{l}\text { Повышение точности моделирования и оптимизация портфеля активов } \\
\text { Рационализация методов контроля и капитальных затрат }\end{array}$ \\
\hline Покорение новых рубежей & $\begin{array}{l}\text { Внедрение технологий дистанционного управления и автономности } \\
\text { Снижение потребности в собственном персонале и подрядчиках }\end{array}$ \\
\hline $\begin{array}{l}\text { «Ресурсный национализм» и экологиче- } \\
\text { ские требования }\end{array}$ & $\begin{array}{l}\text { Повышение безопасности производства } \\
\text { Снижение негативного воздействия на окружающую среду } \\
\text { Максимальное вовлечение заинтересованных сторон - анализ социальных сетей и } \\
\text { других средств коммуникации }\end{array}$ \\
\hline Неопределенность спроса/цены & $\begin{array}{l}\text { Интеграция продаж и производственного планирования для получения максимальных } \\
\text { результатов }\end{array}$ \\
\hline $\begin{array}{l}\text { Управление затратами и контроль де- } \\
\text { нежных потоков }\end{array}$ & $\begin{array}{l}\text { Улучшение интеграции между рудником, ГОКом и сбытовыми операциями } \\
\text { Повышение качества сбора и моделирования данных } \\
\text { Сокращение непроизводительных операций на руднике, удаленный доступ к информа- } \\
\text { ции, рост производительности } \\
\text { Снижение капитальных затрат и операционных расходов } \\
\text { Более качественное управление денежным потоком и оборотным капиталом }\end{array}$ \\
\hline $\begin{array}{l}\text { Конкуренция в привлечении талантли- } \\
\text { вых сотрудников }\end{array}$ & $\begin{array}{l}\text { Более эффективное применение профессиональных знаний для решения задач } \\
\text { Более качественные методы обучения и современная поддержка } \\
\text { Изменение условий труда для привлечения нового поколения сотрудников } \\
\text { Решение проблем, связанных со старением персонала и сохранением знаний }\end{array}$ \\
\hline $\begin{array}{l}\text { Преодоление жизненного цикла ак- } \\
\text { тивов и оборудования с сохранением } \\
\text { операционных затрат на низком уровне }\end{array}$ & $\begin{array}{l}\text { Более длительный срок эксплуатации активов и оборудования, более высокая их рен- } \\
\text { табельность } \\
\text { Повышение доступности оборудования } \\
\text { Повышение безопасности при эксплуатации крупногабаритной техники }\end{array}$ \\
\hline Повышение производительности & $\begin{array}{l}\text { Интеграция всех процессов - от рудника до рынка сбыта } \\
\text { Анализ растущего объема геологических и производственных данных и формирова- } \\
\text { ние рекомендаций, нацеленных на принятие практических решений } \\
\text { Оптимизация имеющихся ресурсов в соответствии с рынком и пропускной способно- } \\
\text { стью транспортировки }\end{array}$ \\
\hline
\end{tabular}

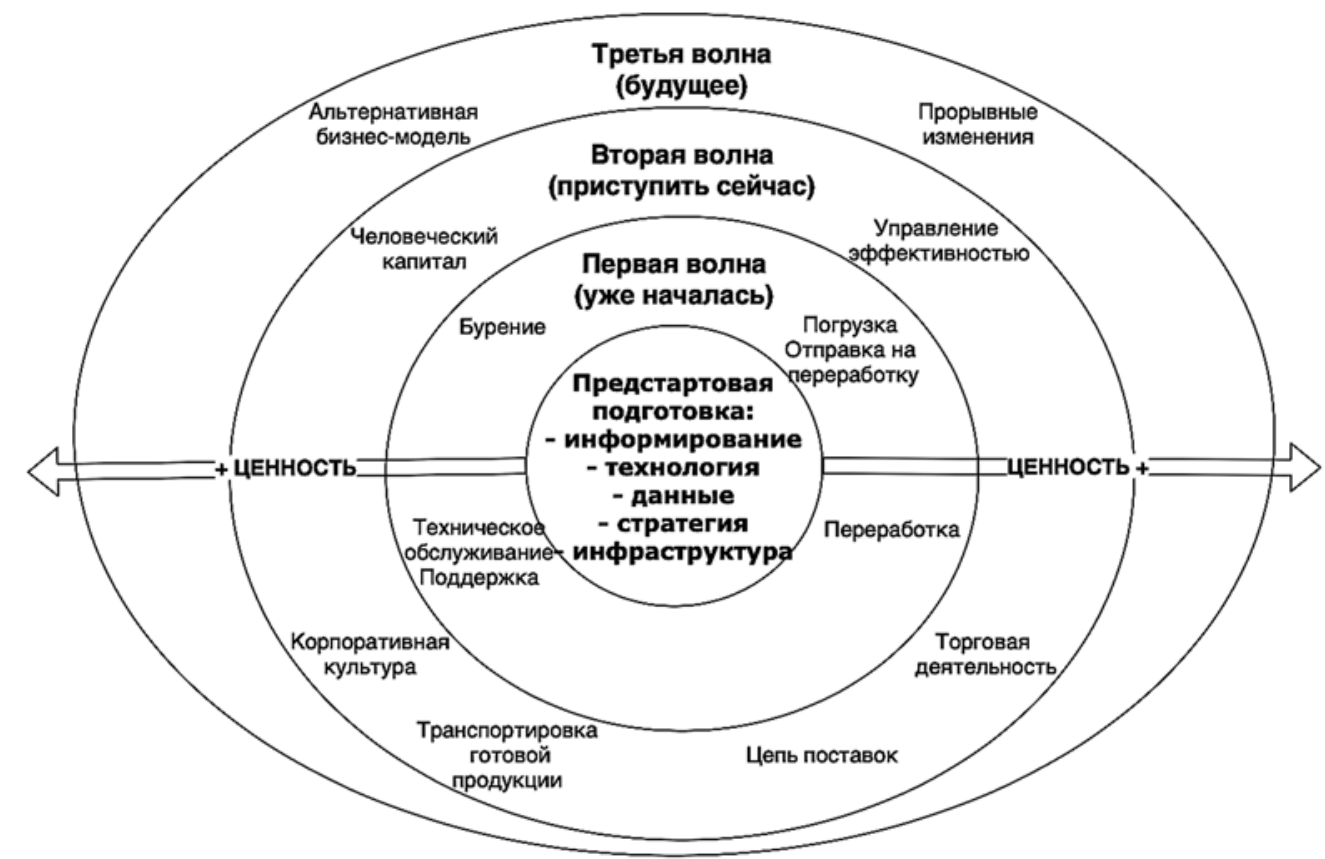

Волновой подход к реализации программы цифровой трансформации горнодобывающих предприятий 


\section{Опыт цифровой трансформации горнодобывающих предприятий}

\begin{tabular}{|c|c|c|c|}
\hline Компания & כтап & Опыт цифровой трансформации & Ожидаемые эффекты \\
\hline \multirow[t]{3}{*}{$\begin{array}{l}\text { Горно- } \\
\text { металлургический } \\
\text { холдинг «ЕВРАЗ» }\end{array}$} & $\begin{array}{l}\text { Базовая } \\
\text { автоматизация }\end{array}$ & $\begin{array}{l}\text { Внедрение автоматизированной системы мони- } \\
\text { торинга горнотранспортной техники в карьерах } \\
\text { КГОКа. Автоматизация процесса продувки стали в } \\
\text { конвертерах ЗСМК. Внедрение системы оптимиза- } \\
\text { ции технологического процесса выплавки чугуна } \\
\text { на доменной печи № } 7 \text { НТМК }\end{array}$ & $\begin{array}{l}\text { Подготовка массивов данных (Big } \\
\text { Data). Совершенствование процес- } \\
\text { сов, обеспечение готовности произ- } \\
\text { водства и поддерживающих функ- } \\
\text { ций к цифровой трансформации }\end{array}$ \\
\hline & Цифровизация & $\begin{array}{l}\text { Запуск мобильных решений для безопасной до- } \\
\text { бычи угля в шахтах. Разработка системы математи- } \\
\text { ческого моделирования производственных пере- } \\
\text { делов на предприятиях в Сибири }\end{array}$ & $\begin{array}{l}\text { Объективный контроль загазован- } \\
\text { ности шахт, состояния выработок, } \\
\text { работы горношахтного оборудо- } \\
\text { вания }\end{array}$ \\
\hline & $\begin{array}{l}\text { Цифровая } \\
\text { трансформация }\end{array}$ & $\begin{array}{l}\text { Применение искусственного интеллекта, разви- } \\
\text { тие экспертных систем, центров диспетчеризации, } \\
\text { сквозного качества и сценарного планирования, } \\
\text { развитие технологий цифровой шахты }\end{array}$ & $\begin{array}{l}\text { Создание качественно иной плат- } \\
\text { формы (Digital Ready) и новых ин- } \\
\text { струментов для принятия управлен- } \\
\text { ческих решений, повышение эффек- } \\
\text { тивности производства }\end{array}$ \\
\hline $\begin{array}{l}\text { Горно- } \\
\text { металлургический } \\
\text { холдинг } \\
\text { «Металлоинвест» }\end{array}$ & $\begin{array}{l}\text { Автоматизация } \\
\text { и цифровизация }\end{array}$ & $\begin{array}{l}\text { Реализуется комплексная программа цифровиза- } \\
\text { ции бизнеса «Industry 4.0», в рамках которой, по- } \\
\text { мимо внедрения на базе ERP-системы SAP запу- } \\
\text { щен ряд проектов, в частности «Обмен электрон- } \\
\text { ным документооборотом», «Быстрое закрытие от- } \\
\text { четного периода», «Договорная работа», «Серви- } \\
\text { сы самообслуживания», «Обеспечение информа- } \\
\text { ционной безопасности» и т.д. }\end{array}$ & $\begin{array}{l}\text { Пересмотр существующих бизнес- } \\
\text { процессов и внедрение новых. Соз- } \\
\text { дание умного и интеллектуального } \\
\text { предприятия, использование пре- } \\
\text { имуществ цифровизации на управ- } \\
\text { ленческом и производственном } \\
\text { уровнях }\end{array}$ \\
\hline \multirow[t]{2}{*}{$\begin{array}{l}\text { ПАО «Южный } \\
\text { Кузбасс» } \\
\text { (входит в группу } \\
\text { «Мечел») }\end{array}$} & \multirow[t]{2}{*}{$\begin{array}{l}\text { Автоматизация, } \\
\text { цифровизация }\end{array}$} & $\begin{array}{l}\text { Формирование единого информационного ланд- } \\
\text { шафта, обеспечивающего интеграцию систем про- } \\
\text { мышленного уровня с бизнес-приложениями }\end{array}$ & $\begin{array}{l}\text { Создание единого цикла планиро- } \\
\text { вания, исполнения, контроля, уче- } \\
\text { та и анализа деятельности, сокраще- } \\
\text { ние времени реакции на изменения }\end{array}$ \\
\hline & & $\begin{array}{l}\text { Применение автоматизированных систем на обо- } \\
\text { гатительных фабриках }\end{array}$ & $\begin{array}{l}\text { Точное дозирование реагентов, ис- } \\
\text { ключение перерасхода, получение } \\
\text { продукции с необходимыми харак- } \\
\text { теристиками качества. Снижение се- } \\
\text { бестоимости готовой продукции, } \\
\text { повышение конкурентоспособности }\end{array}$ \\
\hline $\begin{array}{l}\text { ПАО «Распадская» } \\
\text { (входит в холдинг } \\
\text { «ЕВРАЗ») }\end{array}$ & Цифровизация & $\begin{array}{l}\text { Применение системы беспилотных летательных } \\
\text { аппаратов (дронов) для автоматизации маркшей- } \\
\text { дерской съемки }\end{array}$ & $\begin{array}{l}\text { Сокращение времени простоя ка- } \\
\text { рьерной техники. Более оператив- } \\
\text { ное принятие производственных и } \\
\text { управленческих решений }\end{array}$ \\
\hline $\begin{array}{l}\text { АО «Сибирская } \\
\text { угольная } \\
\text { энергетическая } \\
\text { компания» (СУЭК) }\end{array}$ & Цифровизация & $\begin{array}{l}\text { Буровые станки с помощью датчиков и специаль- } \\
\text { но разработанных алгоритмов выполняют основ- } \\
\text { ные операции, которые в настоящее время кон- } \\
\text { тролируются в ручном режиме. Использование } \\
\text { роботизированных самосвалов в карьерах }\end{array}$ & $\begin{array}{l}\text { Повышение эффективности произ- } \\
\text { водства }\end{array}$ \\
\hline
\end{tabular}

В настоящее время подготовка CDTO ведется рядом учебных заведений. В Московской школе управления «Сколково» открыта дипломная образовательная программа для директоров и команд по цифровой трансформации компаний CDTO. Программы цифровой трансформации уже реализуются на горнодобывающих предприятиях. Флагманом является холдинг «ЕВРАЗ», который с 2017 г. реализует полноценную программу цифровой трансформации по трем секторам: металлургический сегмент, добывающие активы, блок продаж и административные функции. В 2018-2019 гг. реализовано сквозное сценарное планирование всей производственной цепочки «ЕВРАЗ ЗСМК», что позволило получить значительный экономический эффект. Созданы математические модели (так называемые «цифровые двойники») каждого передела с точностью 99\%. Ежегодный эффект от данного проекта состав- ляет свыше 600 млн руб. [13]. В соответствии с волновой концепцией (см. рисунок) ЕВРАЗ реализует третью волну, характеризующуюся самыми сильными рисками.

\section{ЗАКЛЮЧЕНИЕ}

На основании проведенного исследования можно сделать вывод, что цифровая трансформация горнодобывающей промышленности - сложный общественный вызов, который требует договоренностей между ключевыми стейкхолдерами. Реализация программы цифровой трансформации на основе предложенного алгоритма, который успешно внедряется в холдинге «ЕВРАЗ» на заключительном этапе, позволяет повысить эффективность, управляемость и конкурентоспособность горнодобывающей компании, делая ее более гибкой и устойчивой к рыночным изменениям, является ярким примером цифровой трансфор- 
мации для других предприятий данной отрасли. Развитие процессов цифровой трансформации от внедрения цифровых технологий во внутренние бизнес-процессы компании к построению внешних цифровых каналов рыночной коммуникации позволит отечественным горнодобывающим компаниям вести своюдеятельность в максимально открытом для инвесторов информационном режиме.

\section{Список литературы}

1. Digital transformation: A multidisciplinary reflection and research agenda / P.C. Verhoef, T. Broekhuizen, Y. Bart et al. // Journal of Business Research. 2021. Vol. 122. P. 889-901. DOI: 10.1016/j.jbusres.2019.09.022.

2. Плакиткин Ю.А., Плакиткина Л.С. Цифровизация экономики угольной промышленности России - от «Индустрии-4.0» до «Общества 5.0» // Горная Промышленность, 2018. № 4 (140). С. 22-30.

3. Норицугу Уэ. Общество 5.0: взгляд Mitsubishi Electric // Экономические стратегии. 2017. № 4. С. 2-11.

4. Manganello K. Will Industry 5.0 really be revolutionary? URL: https://www.thomasnet.com/insights/will-industry-5-0really-be-revolutionary/ (дата обращения: 15.01.2021).

5. Industrie 4.0 roadmap: Framework for digital transformation based on the concepts of capability maturity and alignment / A. Issa, B. Hatiboglu, A. Bildstein et al. // Procedia CIPR. 2018. Vol. 72. P. 973-978. DOI: 10.1016/j. procir.2018.03.151.

6. Müller E., Hopf H. Competence Center for the Digital Transformation in Small and Medium-Sized Enterprises
// Procedia Manufacturing. 2017. Vol. 11. P. 1495-1500. DOI: 10.1016/j.promfg.2017.07.281.

7. Особенности и тенденции цифровой трансформации российской горнодобывающей отрасли / Д.В. Лютягин, В.П. Яшин, Ю.В. Забайкин и др. // Экономика: вчера, сегодня, завтра. 2019. Т. 9. № 7А. С. 147-159.

8. Степанов И. Цифровизация угля и металла // Коммерсантъ. 2019. № 174. С. 9.

9. Новоселов С.В., Мельник В.В., Агафонов В.В. Экспортно ориентированная стратегия развития угольных компаний России - основной фактор обеспечения их финансовой устойчивости // Уголь. 2017. № 11. С. 54-56. DOI: 10.18796/0041-5790-2017-11-54-56.

10. Новоселов С.В. Альтернативные подходы и дискуссионные вопросы при проектировании шахт нового поколения уровня 2035 года // Уголь. 2019. № 1. С.37-39. DOI: 10.18796/0041-5790-2019-1-37-39.

11. Shinkevich A.I., Kudryavtseva S.S., Ershova I.G. Modelling of energy efficiency factors of petrochemical industry // International Journal of Energy Economics and Policy. 2020. Vol. 10(3). P. 465-470.

12. Шклярук М. Цифровая экономика - командная работа. [Электронный ресурс]. URL: https://drive.google.com/ file/d/1drlbJMIY7ren-6XrU3tN5ZiEccfighP/view (дата обращения: 15.01.2021).

13. Годовой отчет холдинга ЕВРАЗ за 2018 г. [Электронный ресурс]. URL: https://www.evraz.com/upload/iblock/ 9e6/9e61022f8141f31548b6cbd6de6f0ca0.pdf (дата обращения: 15.01.2021).

Original Paper

ECONOMIC OF MINING

UDC 338.45:622.3:517.11.001.57 @ D.Yu. Savon, E.V. Shkarupeta, A.E. Safronov, A.Yu. Anisimov, N.O. Vichrova, 2021

ISSN 0041-5790 (Print) • ISSN 2412-8333 (Online) • Ugol' - Russian Coal Journal, 2021, № 2, pp. 32-37

DOI: http://dx.doi.org/10.18796/0041-5790-2021-2-32-37

Title

Digital transformation of production processes and mining business models in the conditions of market instability

\author{
Authors \\ Savon D.Yu. ${ }^{1}$, Shkarupeta E.V. ${ }^{2}$, Safronov A.E. ${ }^{3}$, Anisimov A.Yu. ${ }^{4}$, Vichrova N.O. ${ }^{1}$ \\ ${ }^{1}$ National University of Science and Technology "MISIS" (NUST "MISIS"), Moscow, 119049, Russian Federation \\ ${ }^{2}$ Voronezh State Technical University, Voronezh, 394071, Russian Federation \\ ${ }^{3}$ Don State Technical University, Rostov-on-Don, 344010, Russian Federation \\ ${ }^{4}$ Institute of world civilizations NANO HE, Moscow, 119991, Russian Federation
}

\section{Authors' Information}

Savon D.Yu., Doctor of Economic Sciences, Professor of Industrial Management department, e-mail: di199@yandex.ru

Shkarupeta E.V., Doctor of Economic Sciences, Professor of Digital and Industrial Economics department, e-mail: 9056591561@mail.ru Safronov A.E., Doctor of Economic Sciences, Professor of Management and business technology department, e-mail: reception@dstu.edu.ru Anisimov A.Yu., PhD (Economic), Associate Professor of Finance and credit department, e-mail: anisimov_au@mail.ru

Vichrova N.O., PhD (Economic), Associate Professor of Economics department, e-mail: natalia.vichrova@yandex.ru

\section{Abstract}

The digital transformation of production processes and business models of mining enterprises, going hand in hand with the selection of appropriate digital technologies, will be able to provide long-term solutions to the alarming economic problems of our time. The article raises issues related to the current and future state of the mining industry before, during and after the digital transformation. The digital transformation of mining enterprises by the authors of the article means not only the process of integrating digital technologies into all aspects of the mining industry (which, in essence, is digitalization), but also introducing fundamental changes in technologies, culture, operations and principles for creating new products and services in the industry. The study examined the need, benefits, content, goals and objectives, principles, main directions, tools and stages of the digital transformation of mining enterprises. The systemic problems of digital transformation of the industry are identified, on the basis of which the authors proposed an algorithm for digital transformation of mining enterprises with step-by-step implementation, which has already begun to be applied in the EVRAZ holding. In the process of digital transformation, a special role is assigned to the head of digital transformation (CDTO). The presented developments will make it possible to comprehensively transform the domestic mining industry on the basis of a change in strategy and the application of new operating business models at the global level 
in the conditions of market instability, volatile demand, problems of finding new deposits in new regions, the need for longer-term exploitation of assets, focus on high standards in work, as well as changes in the rules of conducting mining business around the world.

\section{Keywords}

Digital transformation, Digitalization, Mining, Mining enterprises, Mining industry, Chief digital transformation officer, CDTO.

\section{References}

1. Verhoef P.C., Broekhuizen T., Bart Y. et al. Digital transformation: A multidisciplinary reflection and research agenda. Journal of Business Research, 2021, Vol. 122. pp. 889-901. DOI: 10.1016/j.jbusres.2019.09.022.

2. Plakitkin Yu.A. \& Plakitkina L.S. Digitalization of Russian Coal Industry Economy: from Industry 4.0 to Society 5.0. Gornaâ promyshlennost', 2018, (4), pp. 22-30. (In Russ.).

3. Noritsugu U. Society 5.0: the View of Mitsubishi Electric. Ekonomicheskie strategii, 2017, (4), pp. 2-11. (In Russ.).

4. Manganello K. Will Industry 5.0 really be revolutionary? Available at: https:// www.thomasnet.com/insights/will-industry-5-0-really-be-revolutionary/ (accessed 15.01.2021).

5. Issa A., Hatiboglu B., Bildstein A. et al. Industrie 4.0 roadmap: Framework for digital transformation based on the concepts of capability maturity and alignment. Procedia CIPR, 2018, Vol. 72, pp. 973-978. DOI: 10.1016/j.procir.2018.03.151.

6. Müller E. \& Hopf H. Competence Center for the Digital Transformation in Small and Medium-Sized Enterprises. Procedia Manufacturing, 2017, Vol. 11, pp. 1495-1500. DOI: 10.1016/j.promfg.2017.07.281.

7. Lyutyagin D.V., Yashin V.P., Zabaykin Yu.V. et al. Specific features and trends in digital transformation of the Russian mining industry. Ekonomika: vchera, segodnya, zavtra, 2019, Vol. 9, No. 7A, pp. 147-159. (In Russ.).
8. Stepanov I. Digitalization of coal and metal. Kommersant, 2019, (174), pp. 9. (In Russ.).

9. Novoselov S.V., Melnik V.V. \& Agafonov V.V. Export-oriented development strategy of the coal companies of Russia - the main factor ensuring their financial stability. Ugol', 2017, (11), pp. 54-56. (In Russ.). DOI: 10.18796/00415790-2017-11-54-56.

10. Novoselov S.V. Alternative approaches and controversial issues in the design of new mines generation level 2035. Ugol', 2019, (1), pp. 37-39. (In Russ.). DOI: 10.18796/0041-5790-2019-1-37-39.

11. Shinkevich A.I., Kudryavtseva S.S. \& Ershova I.G. Modelling of energy efficiency factors of petrochemical industry. International Journal of Energy Economics and Policy, 2020, Vol. 10(3), pp. 465-470.

12. Shklyaruk M. Digital economy: a team effort. [Electronic resource]. Available at: https://drive.google.com/file/d/1drlbJMIY7ren-6XrU3tN5ZiEccfighP/ view (accessed 15.01.2021). (In Russ.).

13. EVRAZ Annual Report \& Accounts, 2018 [Electronic resource]. Available at: https://www.evraz.com/upload/iblock/9e6/9e61022f8141f31548b6cbd6 de6f0ca0.pdf (accessed 15.01.2021).

\section{For citation}

Savon D.Yu., Shkarupeta E.V., Safronov A.E., Anisimov A.Yu. \& Vichrova N.O. Digital transformation of production processes and mining business models in the conditions of market instability. Ugol', 2021, (2), pp. 32-37. (In Russ.). DOI: 10.18796/0041-5790-2021-2-32-37.

\section{Paper info}

Received October 23, 2020

Reviewed November 19, 2020

Accepted January 12, 2021 